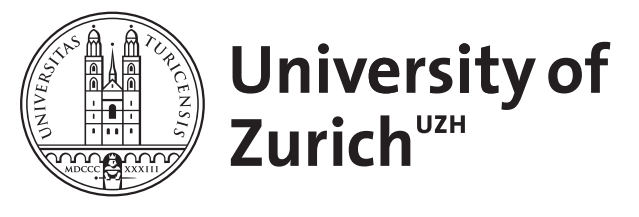

\title{
Inertial forces and photon surfaces in arbitrary spacetimes
}

\author{
Foertsch, T ; Hasse, W ; Perlick, V
}

\begin{abstract}
Given, in an arbitrary spacetime (M, g), a two-dimensional timelike submanifold $\Sigma$ and an observer field $\mathrm{n}$ on $\Sigma$, we assign gravitational, centrifugal, Coriolis and Euler forces to every particle worldline in $\Sigma$ with respect to $\mathrm{n}$. We prove that centrifugal and Coriolis forces vanish, for all in $\Sigma$ with respect to any $n$, if and only if $\Sigma$ is a photon 2 -surface, i.e., generated by two families of lightlike geodesics. We further demonstrate that a photon 2-surface can be characterized in terms of gyroscope transport and we give several mathematical criteria for the existence of photon 2-surfaces. Finally, examples of photon 2-surfaces in conformally flat spacetimes, in Schwarzschild and Reissner-Nordström spacetimes, and in Gödel spacetime are worked out.
\end{abstract}

DOI: https://doi.org/10.1088/0264-9381/20/21/006

Posted at the Zurich Open Repository and Archive, University of Zurich

ZORA URL: https://doi.org/10.5167/uzh-21872

Journal Article

Accepted Version

Originally published at:

Foertsch, T; Hasse, W; Perlick, V (2003). Inertial forces and photon surfaces in arbitrary spacetimes. Classical and Quantum Gravity, 20(21):4635-4651.

DOI: https://doi.org/10.1088/0264-9381/20/21/006 


\title{
Inertial forces and photon surfaces in arbitrary spacetimes
}

\author{
Thomas Foertsch* Wolfgang Hasse ${ }^{\dagger} \quad$ Volker Perlick $^{\ddagger}$
}

\begin{abstract}
Given, in an arbitrary spacetime $(M, g)$, a 2-dimensional timelike submanifold $\Sigma$ and an observer field $n$ on $\Sigma$, we assign gravitational, centrifugal, Coriolis and Euler forces to every particle worldline $\lambda$ in $\Sigma$ with respect to $n$. We prove that centrifugal and Coriolis forces vanish, for all $\lambda$ in $\Sigma$ with respect to any $n$, if and only if $\Sigma$ is a photon 2 -surface, i.e., generated by two families of lightlike geodesics. We further demonstrate that a photon 2-surface can be characterized in terms of gyroscope transport and we give several mathematical criteria for the existence of photon 2-surfaces. Finally, examples of photon 2-surfaces in conformally flat spacetimes, in Schwarzschild and Reissner-Nordström spacetimes, and in Gödel spacetime are worked out.
\end{abstract}

PACS numbers: $04.20 \mathrm{Cv} 02.40 \mathrm{Hw}$

\section{Introduction}

In this paper we discuss the question of how to define inertial forces in an arbitrary general-relativistic spacetime, i.e., in a Lorentzian manifold $(M, g)$. Given a particle's worldline $\lambda$ in $M$, the 4-acceleration of $\lambda$ is unambiguously defined. The negative 4-acceleration of $\lambda$ gives the acceleration of a freely falling particle relative to $\lambda$. After multiplication with the freely falling particle's mass we get the total "apparent force" that acts, in the view of $\lambda$, upon the freely falling particle. By the question of how to define inertial forces we mean the question of how to decompose this total "apparent force" into gravitational, centrifugal, Coriolis and Euler force. This decomposition requires some additional information, i.e., it cannot be done in an absolute way. (The statement that the

\footnotetext{
*Mathematisches Institut, Universität Zürich-Irchel, 8057 Zürich, Switzerland. Email: foertsch@math.unizh.ch

†Institut für Theoretische Physik, TU Berlin, Sekr. PN 7-1, 10623 Berlin, Germany, and Wilhelm-Foerster-Sternwarte Berlin, 12169 Berlin, Germany. Email: astrometrie@gmx.de

${ }^{\ddagger}$ Institut für Theoretische Physik, Universität zu Köln, 50923 Köln, Germany. Permanent address: Institut für Theoretische Physik, TU Berlin, Sekr. PN 7-1, 10623 Berlin, Germany. Email: vper0433@itp.physik.tu-berlin.de
} 
gravitational force has no absolute meaning is one version of the equivalence principle.) In a static or stationary spacetime, this additional information is provided by the timelike Killing vector field which, after normalization, can be interpreted as the 4 -velocity field of a distinguished family of observers. With respect to this distinguished observer field, inertial forces can be uniquely assigned to each particle's worldline, as was discussed by Abramowicz and collaborators in various articles, see, e.g. Abramowicz, Carter and Lasota [1], Abramowicz and Prasanna [2] and Abramowicz [3]. They also provided a geometric interpretation of inertial forces in terms of the so-called reference-3-geometry that was obtained by conformally adjusting the quotient-space metric. For a discussion of these ideas we also refer to a forthcoming book by Abramowicz and Sonego [4].

The results in static and stationary spacetimes led Abramowicz and others to seek for particular observer fields in general spacetimes that allowed to define similar reference-3-geometries and thus to generalize the notion of inertial forces to general spacetimes. However, their suggested generalizations (see Abramowicz, Nurowski and Wex [5]) have been partially criticized by several authors (see for example de Felice [6], Sonego and Massar [7] and Bini, Carini and Jantzen [8]). Some of the critical comments refer to the fact that the inertial forces, as defined in [5], have unwanted features, others to the fact that they are in a certain sense ambiguous. Thus, it seems fair to say that the appropriate definition of inertial forces in arbitrary spacetimes is still a matter of debate.

In this paper we turn away from the idea that in arbitrary spacetimes inertial forces should be defined with respect to an observer field with particular properties. We rather define inertial forces with respect to arbitrary timelike surfaces $\Sigma$ and arbitrary observer fields given on $\Sigma$. More precisely, we choose, in an arbitrary spacetime $(M, g)$, a timelike surface $\Sigma$ and a vector field $n$ on $\Sigma$ that is normalized to $g(n, n)=-1$. We demonstrate that then gravitational, centrifugal, Coriolis and Euler forces can be unambiguously assigned to any particle's worldline $\lambda$ contained in $\Sigma$. These definitions will be given in Section 2 below.

For the sake of illustration, one may interpret $\Sigma$ as the worldsheet of a roller-coaster track and $n$ as the 4 -velocity field of observers distributed along the track. Then the worldlines $\lambda$ contained in $\Sigma$ can be associated with the motions allowed for cars that are bound to the roller-coaster track but are free to move arbitrarily along the track. This roller-coaster interpretation, which was used e.g. by Abramowicz in [3], is quite helpful for associating inertial forces with physical intuition. However, we emphasize that the roller-coaster interpretation is only a didactical means. For our mathematical results to be true it is not necessary to associate $\Sigma$ with a material object.

The idea of defining the centrifugal force with respect to a prescribed spatial track can be traced back to Huygens, whereas the definition usually given in mechanics text-books is due to Newton, see Abramowicz [3], p.735/736. Our definitions of inertial forces in Section 2 can be viewed as a literal adaptation of Huygens' definition of centrifugal force to general relativity. We share Abramowicz's view that, in the context of general relativity, Newton's definition is not 
appropriate.

If a timelike vector field $K$ has been chosen on $(M, g)$, every timelike curve $\lambda$ that is nowhere tangent to $K$ naturally defines a timelike surface $\Sigma$, namely the set of all points that can be connected to $\lambda$ by an integral curve of $K$. Restricting to $\Sigma$ and normalizing $K$ gives a vector field $n$ on $\Sigma$. In other words, once a timelike vector field $K$ has been chosen on $(M, g)$ we have a distinguished choice for $(\Sigma, n)$ and, thus, unique inertial forces for every worldline $\lambda$ that is nowhere tangent to $K$. (For a $\lambda$ that is tangent to $K$, at some points or everywhere, we may choose any timelike surface $\Sigma$ that contains $\lambda$ and is invariant under the flow of $K$; the inertial forces defined along $\lambda$ turn out to be the same for all these choices.) The crucial point is that $K$ need not satisfy any additional condition. In the special case that $K$ is a Killing vector field our definition of inertial forces reduces to the generally accepted one in static or stationary spacetimes.

Having defined inertial forces with respect to a timelike surface $\Sigma$, it is an interesting problem to determine those $\Sigma$ for which centrifugal and Coriolis forces vanish. We do this in Section 3 by proving the

Theorem 1. Let $(M, g)$ be an arbitrary spacetime and $\Sigma$ a 2-dimensional timelike submanifold of $M$. Then the following properties are mutually equivalent:

(i) For every particle worldline $\lambda$ contained in $\Sigma$ the centrifugal and Coriolis forces with respect to any vector field $n$ on $\Sigma$ with $g(n, n)=-1$ vanish.

(ii) $\Sigma$ is generated by two congruences of lightlike geodesics.

(iii) If $n$ and $\tau$ are any two vector fields on $\Sigma$ with $g(\tau, \tau)=-g(n, n)=1$ and $g(n, \tau)=0$, the vector field $\tau$ is Fermi-Walker-parallel along $n$, i.e., $\nabla_{n} \tau$ is a multiple of $n$.

If we interpret $\Sigma$ as the worldsheet of a track, Property (ii) means that the track appears straight to the eye of an observer who looks ("backward" or "forward") along the track. As, according to general relativity, a gyroscope always remains Fermi-Walker-parallel to itself (see, e.g. Misner, Thorne and Wheeler [9], Sect. 40.7), Property (iii) means that when transported along the track a gyroscope whose axis is initially parallel to the track always stays parallel to the track.

Note that, although the centrifugal and Coriolis forces depend on the choice of $n$, Theorem 1 demonstrates that the condition of vanishing centrifugal and Coriolis forces is independent of $n$, i.e., it is a geometric property of $\Sigma$. Moreover, Theorem 1 implies that properties (i) and (iii) are conformally invariant because property (ii) obviously is. The conformal invariance of property (iii) reflects the known fact that the notion of an "inertial compass" is conformally invariant.

A trivial example for a timelike surface $\Sigma$ that obviously satisfies Property (ii) and, thus, Properties (i) and (iii) of Theorem 1 is a timelike plane in Minkowski spacetime. The best known non-trivial example is the surface $r=3 M, \theta=\pi / 2$ in the Schwarzschild spacetime. If we interpret this surface as the worldsheet of a track, someone moving along this track feels neither centrifugal nor Coriolis forces, although the track is circular; at the same time, 
Properties (ii) and (iii) demonstrate that the track appears straight to the eye and turns out to be straight if deviation from straightness is measured by transporting a gyroscope.

In generalizing this well-known Schwarzschild example, Abramowicz [3] has considered timelike surfaces that are invariant under the flow of the Killing vector fields $\partial_{t}$ and $\partial_{\varphi}$ in a static axisymmetric spacetime. He has shown that in this case Property (i) of Theorem 1 implies Properties (ii) and (iii). Our result generalizes this observation to arbitrary timelike surfaces in arbitrary spacetimes.

Theorem 1 gives three equivalent characterizations of surfaces but it does not say anything about the existence of such surfaces in arbitrary spacetimes. We call a 2-dimensional timelike submanifold $\Sigma$ that satisfies Property (ii) and, thus, Properties (i) and (iii) of Theorem 1 a timelike photon 2-surface. A similar notion was used by Claudel, Virbhadra and Ellis [10]. In arbitrary spacetimes, the existence of timelike photon 2-surfaces is not guaranteed. Some existence criteria and construction methods are presented in Section 4. Finally in Section 5 we give various examples for timelike photon 2 -surfaces.

\section{The definition of inertial forces}

In this section we define the inertial forces in an arbitrary spacetime. Here, by a spacetime we mean any finite-dimensional Lorentzian manifold $(M, g)$. For physical reasons, $(M, g)$ should be 4-dimensional, time-orientable, and connected; mathematically, however, we shall not need these particular assumptions. Our results are non-trivial only if $M$ is at least 3-dimensional.

Throughout this paper, our conventions are as follows. We use physical units making the velocity of light equal to one and our sign convention for the Lorentzian signature is $(+, \ldots,+,-)$. The Levi-Civita derivative of $g$ is denoted by $\nabla$. All (sub)manifolds and maps are tacitly assumed to be sufficiently often differentiable such that the written derivatives exist. By a submanifold we mean what is more fully called an immersed submanifold, as opposed to an imbedded submanifold, i.e., we allow for self-intersections. This is irrelevant for the definition of inertial forces, which is purely local, and for the three conditions in Theorem 1, whose equivalence can be verified for arbitrarily small pieces of $\Sigma$; however, it is important to note that our construction methods for photon surfaces may yield surfaces with self-intersections. For a submanifold $\Sigma$, we distinguish between vector fields on $\Sigma$ and vector fields along $\Sigma$. A vector field on $\Sigma$ assigns to each point $p \in \Sigma$ a vector in the tangent space $T_{p} \Sigma$ whereas a vector field along $\Sigma$ assigns to $p \in \Sigma$ a vector in the tangent space $T_{i(p)} M$ where $i$ denotes the immersion $\Sigma \rightarrow M$. (An analogous terminology applies to tensor fields of higher rank.) To make the distinction easily seen we use lower-case letters for vector fields on a submanifold of $M$ and upper-case letters for vector fields that take values in the full tangent space of $M$.

What we need in order to define the inertial forces is a pair $(\Sigma, n)$, where $\Sigma$ is a 2-dimensional timelike submanifold of $M$, and $n$ is a vector field on $\Sigma$ 
with $g(n, n)=-1$. Given any timelike curve $\lambda: I_{\lambda} \longrightarrow \Sigma$ whose tangent vector field $u=\dot{\lambda}$ satisfies the usual 4-velocity normalization condition $g(u, u)=-1$, it is our goal to assign inertial accelerations to $\lambda$ with respect to $(\Sigma, n)$. The inertial forces acting upon a particle are then given by multiplying the inertial accelerations with the particle's mass.

With $(\Sigma, n)$ given, the conditions

$$
g(n, \tau)=0 \quad \text { and } \quad g(\tau, \tau)=1
$$

define a vector field $\tau$ on $\Sigma$ uniquely up to sign. After fixing $\tau$ by choosing one of the two signs, we can decompose the 4-velocity $u$ of each worldline $\lambda$ in the form

$$
u=\gamma(n+v \tau), \quad \gamma=\frac{1}{\sqrt{1-v^{2}}} .
$$

This equation defines the spatial velocity $v$ of $\lambda$ with respect to $n$. Mathematically, $v$ is a map that assigns to the curve parameter a number between -1 and 1. From (2) we can calculate the 4 -acceleration $\nabla_{u} u$ of $\lambda$,

$$
\begin{array}{r}
\nabla_{u} u=\nabla_{n} n+\gamma^{2} v\left(\nabla_{n} \tau+\nabla_{\tau} n\right)+ \\
\gamma^{2} v^{2}\left(\nabla_{\tau} \tau+\nabla_{n} n\right)+\nabla_{u}(\gamma v) \tau+\left(\nabla_{u} \gamma\right) n .
\end{array}
$$

As $\nabla_{u} u$ gives the acceleration of $\lambda$ relative to freely falling particles, relative to $\lambda$ a freely falling particle is subject to the "inertial acceleration" $a=-\nabla_{u} u$. The gravitational, centrifugal, Coriolis and Euler accelerations with respect to the observer field $n$ are obtained by the following unique decomposition of $a$ (and multiplication with the freely falling particle's mass gives the respective forces).

(a) We decompose $a$ into a component orthogonal to $n$ and a component parallel to $n$. By (3), the component orthogonal to $n$ is

$$
\begin{array}{r}
a+g(a, n) n=-\nabla_{n} n-\gamma^{2} v\left(\nabla_{n} \tau+\nabla_{\tau} n+g\left(\nabla_{n} \tau, n\right) n\right)- \\
\gamma^{2} v^{2}\left(\nabla_{\tau} \tau+\nabla_{n} n+g\left(\nabla_{\tau} \tau, n\right) n\right)-\left(\nabla_{u}(\gamma v)\right) \tau .
\end{array}
$$

(b) The gravitational acceleration $a_{\text {gra }}$ is by definition the part of $a+g(a, n) n$ that is independent of $v$, i.e., the term on the right-hand side of (4) that survives if $v$ is identically zero,

$$
a_{\mathrm{gra}}=-\nabla_{n} n .
$$

(c) The remaining part $a+g(a, n) n+\nabla_{n} n$ will be decomposed into the following pieces:

(i) The part orthogonal to $\tau$ and odd in $v$ is the Coriolis acceleration

$$
a_{\text {Cor }}=-\gamma^{2} v\left(\nabla_{n} \tau+\nabla_{\tau} n+g\left(\nabla_{n} \tau, n\right) n-g\left(\nabla_{\tau} n, \tau\right) \tau\right) .
$$


(ii) The part orthogonal to $\tau$ and even in $v$ is the centrifugal acceleration

$$
a_{\mathrm{cen}}=-\gamma^{2} v^{2}\left(\nabla_{\tau} \tau+\nabla_{n} n+g\left(\nabla_{\tau} \tau, n\right) n-g\left(\nabla_{n} n, \tau\right) \tau\right) .
$$

(iii) The part parallel to $\tau$ is the Euler acceleration

$$
a_{\mathrm{Eul}}=-\left(\nabla_{u}(\gamma v)-\gamma^{2} v g\left(\nabla_{\tau} \tau, n\right)+\gamma^{2} v^{2} g\left(\nabla_{n} n, \tau\right)\right) \tau .
$$

Note that this decomposition ensures that two worldlines $\lambda_{1}$ and $\lambda_{2}$ with $\lambda_{1}(0)=$ $\lambda_{2}(0)=p$ and $v_{1}(0)=-v_{2}(0)$ have the same centrifugal accelerations but opposite Coriolis accelerations at $p$.

Whereas $a_{\text {cen }}$ and $a_{\text {Cor }}$ are always perpendicular to $\Sigma$ and $a_{\text {Eul }}$ is always parallel to $\Sigma$, the gravitational acceleration has, in general, a component perpendicular and a component parallel to $\Sigma$, say $a_{\text {gra }}=a_{\text {gra }}^{\perp}+a_{\text {gra }}^{\|}$. Thus, the components of $a$ perpendicular and parallel to $\Sigma$ are, respectively,

$$
a^{\perp}=a_{\text {gra }}^{\perp}+a_{\text {cen }}+a_{\text {Cor }}, \quad a^{\|}=a_{\text {gra }}^{\|}+a_{\text {Eul }}-g(a, n) n .
$$

Clearly, $a^{\perp}$ and $a^{\|}$are invariant with respect to changing the observer field $n$. As $a=-\nabla_{u} u$ is orthogonal to $u$, both $a^{\perp}$ and $a^{\|}$are spatial vectors in the local rest system of the particle $\lambda$. If we interpret $\Sigma$ as the worldsheet of a track, $a^{\perp}$ gives the inertial acceleration perpendicular to the track whereas $a^{\| l}$ gives the inertial acceleration parallel to the track. The latter involves a derivative of the velocity that enters into the Euler force; hence, it depends on whether the particle accelerates or decelerates in the direction of the track. As a consequence, there is no general relation between $a^{\|}$and the geometry of $\Sigma$. For our Theorem 1, and other results that relate the inertial forces to the geometry of $\Sigma$, it is only $a^{\perp}$ that matters. Note that, since $a_{\text {cen }}$ and $a_{\text {Cor }}$ are spatial vectors in the local rest system of the particle $\lambda$, our Theorem 1 is directly related to inertial accelerations which can be measured in this system.

Recall that, with $(\Sigma, n)$ given, $\tau$ was determined by (1) uniquely up to sign. If we change $\tau$ into $-\tau,(2)$ shows that $v$ changes into $-v$. With this observation it is obvious from (5), (6), (7) and (8) that $a_{\text {gra }}, a_{\text {Cor }}, a_{\text {cen }}$ and $a_{\text {Eul }}$ are indeed uniquely determined by $(\Sigma, n)$ for each worldline $\lambda$ in $\Sigma$. This is an important difference in comparison to the work of Abramowicz, Nurowski and Wex [5] where $\tau$ was uniquely determined only along $\lambda$ and an ambiguity in the definition of inertial forces arose from the necessity of extending $\tau$ off $\lambda$. Another important difference is in the fact that the centrifugal force defined in [5] need not be perpendicular to the plane spanned by the particle's 4-velocity and the observer field.

\section{A characterization of worldsheets for which centrifugal and Coriolis forces vanish}

It is the main purpose of this section to prove Theorem 1 which was announced in the Introduction. To that end we have to introduce some notation. Given 
a 2-dimensional timelike submanifold $\Sigma$ in our Lorentzian manifold $(M, g)$, we decompose at each point $p \in \Sigma$ the tangent space $T_{p} M$ into $T_{p} \Sigma$ and its orthocomplement $\left(T_{p} \Sigma\right)^{\perp}$. With $p$ running over $\Sigma$, the orthogonal projections

$$
P_{p}^{\perp}: T_{p} M \longrightarrow\left(T_{p} \Sigma\right)^{\perp},
$$

define a tensor field $P^{\perp}$ along $\Sigma$ that maps vector fields along $\Sigma$ to vector fields along $\Sigma$. After choosing a vector field $n$ with $g(n, n)=-1$ on $\Sigma$ and denoting by $\tau$ the vector field on $\Sigma$ that is determined by (1) uniquely up to sign, $P^{\perp}$ takes the form

$$
P^{\perp}(Y)=Y-g(\tau, Y) \tau+g(n, Y) n,
$$

where $Y$ is an arbitrary vector field along $\Sigma$. The Coriolis acceleration (6) and the centrifugal acceleration (7) can be rewritten, with the help of $P^{\perp}$, in the following way.

$a_{\mathrm{Cor}}=-\gamma^{2} v P^{\perp}\left(\nabla_{n} \tau+\nabla_{\tau} n\right) \quad$ and $\quad a_{\text {cen }}=-\gamma^{2} v^{2} P^{\perp}\left(\nabla_{n} n+\nabla_{\tau} \tau\right)$.

Since $n$ and $\tau$ are orthonormal, the equation

$$
l^{ \pm}=n \pm \tau
$$

defines two lightlike vector fields $l^{+}$and $l^{-}$on $\Sigma$. At each point $p$ of $\Sigma$, the vectors $l_{p}^{+}$and $l_{p}^{-}$span the two different lightlike lines tangent to $\Sigma$. Note that $l^{+}$and $l^{-}$satisfy

$$
g\left(l^{+}, l^{-}\right)=-2 .
$$

If $n$ has been chosen, $\tau$ is unique up to $\operatorname{sign}$, so $l^{+}$and $l^{-}$are unique up to interchanging. If we replace $n$ by some other vector field $\tilde{n}$ on $\Sigma$ with $g(\tilde{n}, \tilde{n})=$ -1 (and $\tau$ correspondingly by $\tilde{\tau}$ ), the vector fields $l^{+}$and $l^{-}$transform according to

$$
l^{+} \longmapsto A l^{+} \text {and } l^{-} \longmapsto A^{-1} l^{-},
$$

where $A$ is a nowhere vanishing scalar function on $\Sigma$. With the help of (13), the expression (11) for the projection tensor field $P^{\perp}$ can be rewritten as

$$
P^{\perp}(Y)=Y+\frac{1}{2} g\left(l^{-}, Y\right) l^{+}+\frac{1}{2} g\left(l^{+}, Y\right) l^{-} .
$$

As $l^{+}$and $l^{-}$are lightlike, (16) immediately implies

$$
P^{\perp}\left(\nabla_{l^{ \pm}} l^{ \pm}\right)=\nabla_{l^{ \pm}} l^{ \pm}+\frac{1}{2} g\left(l^{\mp}, \nabla_{l^{ \pm}} l^{ \pm}\right) l_{ \pm} .
$$

After these preparations it is now easy to prove the following proposition which will then be used to prove Theorem 1 .

Proposition 1. Let $(M, g)$ be an arbitrary spacetime and $\Sigma$ a 2-dimensional timelike submanifold of $M$. For any choice of a vector field $n$ on $\Sigma$ with $g(n, n)=$ -1 , the equation

$$
a_{\text {cen }}=\mp v a_{\text {Cor }}
$$

holds for all particle worldlines $\lambda$ in $\Sigma$ with the upper sign (or with the lower sign, resp.) if and only if the integral curves of $l^{+}$(or of $l^{-}$, resp.) are geodesics. 
Proof. From (12) and (13) we read

$$
a_{\text {cen }} \pm v a_{\text {Cor }}=-\gamma^{2} v^{2} P^{\perp}\left(\nabla_{l^{ \pm}} l^{ \pm}\right) .
$$

If the integral curves of $l^{ \pm}$are geodesics, $\nabla_{l^{ \pm}} l^{ \pm}$is a multiple of $l^{ \pm}$and, thus, tangent to $\Sigma$, so the right-hand side of (19) vanishes. Hence, the left-hand side has to vanish. Conversely, assume that the left-hand side of (19) vanishes for all particle worldlines in $\Sigma$. Then the right-hand side of (19) has to vanish for all allowed $v$, i.e., $P^{\perp}\left(\nabla_{l^{ \pm}} l^{ \pm}\right)$has to vanish. By (17), this implies that $\nabla_{l^{ \pm}} l^{ \pm}$ is a multiple of $l^{ \pm}$.

As the proof of Proposition 1 is purely algebraic, we have actually proven that (18) holds at a particular point $p$ of $\Sigma$, for any choice of $n$ and for all particle worldlines passing through $p$, if and only if $\nabla_{l^{ \pm}} l^{ \pm}$is a multiple of $l^{ \pm}$at this particular point $p$.

With Proposition 1 at hand, we can now prove our main theorem.

Proof of Theorem 1. Proposition 1 implies that the integral curves of both $l^{+}$ and $l^{-}$are geodesics if and only if (18) holds with both signs for all particle worldlines $\lambda$ in $\Sigma$. The latter condition is, of course, true if and only if $a_{\text {cen }}$ and $a_{\text {Cor }}$ vanish for all particle worldlines $\lambda$ in $\Sigma$. This proves the equivalence of (i) and (ii) in Theorem 1. - To prove the equivalence of (ii) and (iii), we start from the equation

$$
4 \nabla_{n} \tau=\nabla_{l^{+}} l^{+}-\nabla_{l^{-}} l^{-}-\left[l^{+}, l^{-}\right],
$$

which follows directly from (13). As with $l^{+}$and $l^{-}$also their Lie bracket must be tangent to $\Sigma$, this implies

$$
4 P^{\perp}\left(\nabla_{n} \tau\right)=P^{\perp}\left(\nabla_{l^{+}} l^{+}-\nabla_{l^{-}} l^{-}\right) .
$$

When changing to new orthonormal vector fields $\tilde{n}$ and $\tilde{\tau}$, the vector fields $l^{+}$ and $l^{-}$transform according to (15), so

$$
4 P^{\perp}\left(\nabla_{\tilde{n}} \tilde{\tau}\right)=A^{2} P^{\perp}\left(\nabla_{l^{+}} l^{+}\right)-A^{-2} P^{\perp}\left(\nabla_{l^{-}} l^{-}\right) .
$$

Owing to the normalization condition $g(\tilde{\tau}, \tilde{\tau})=1$, the vector field $\nabla_{\tilde{n}} \tilde{\tau}$ is automatically perpendicular to $\tilde{\tau}$; hence, this vector field is parallel to $\tilde{n}$ if and only if it is annihilated by $P^{\perp}$. As a consequence, Property (iii) of Theorem 1 is satisfied if and only if the right-hand side of (22) gives zero for all nowhere vanishing functions $A$ on $\Sigma$. By (17), this is true if and only if the integral curves of $l^{+}$and $l^{-}$are geodesics.

In an arbitrary spacetime, it is easy to construct a timelike surface $\Sigma$ such that the integral curves of either $l^{+}$or $l^{-}$are geodesics: Just choose any lightlike and geodesic vector field $L$ on $M$ and a curve $\lambda$ which is nowhere orthogonal to $L$; then let $\Sigma$ be the set of all points in $M$ that can be connected to $\lambda$ by an integral curve of $L$. By construction, $\Sigma$ is a 2-dimensional immersed timelike submanifold of $M$ near $\lambda$ which is ruled by a congruence of geodesics, namely by integral curves of $L$. The other lightlike congruence in $\Sigma$, however, will not be geodesic in general. In the next section we give criteria for the existence of timelike surfaces for which both $l^{+}$and $l^{-}$are geodesic. 


\section{The existence of photon surfaces}

We begin with the following definition.

Definition 1. A $k$-dimensional submanifold $\Sigma$ of a Lorentzian manifold $(M, g)$ is called a photon $k$-surface if $\Sigma$ is nowhere spacelike and if for every $p \in \Sigma$ and every lightlike vector $w \in T_{p} \Sigma$ the geodesic $\left.\mu:\right]-\varepsilon, \varepsilon[\longrightarrow M$ with $\dot{\mu}(0)=w$ is contained in $\Sigma$, for some $\varepsilon>0$.

This definition slightly modifies the notion of "photon surfaces" as it was extensively discussed by Claudel, Virbhadra and Ellis [10]. The modification is in the fact that Claudel, Virbhadra and Ellis consider only hypersurfaces, i.e., submanifolds of codimension one.

It is obvious from Definition 1 that a 2-dimensional timelike submanifold is a photon 2-surface if and only if it is generated by two families of lightlike geodesics. Thus, Theorem 1 provides two additional characterizations of timelike photon 2-surfaces, as an alternative to the one by lightlike geodesics .

In this section we want to present some general results that guarantee the existence of timelike photon 2-surfaces in some classes of spacetimes and tell us how to construct them. A quite obvious criterion is formulated in the following proposition.

Proposition 2. A point $p$ in a Lorentzian manifold $(M, g)$ admits a neighborhood that can be foliated into timelike photon 2-surfaces if and only if on some neighborhood of $p$ there are two linearly independent lightlike and geodesic vector fields $L^{+}$and $L^{-}$such that the Lie bracket $\left[L^{+}, L^{-}\right]$is a linear combination of $L^{+}$and $L^{-}$. In this case, the photon 2-surfaces are the integral manifolds of the 2-spaces spanned by $L^{+}$and $L^{-}$.

Proof. Two linearly independent lightlike vector fields $L^{+}$and $L^{-}$span a timelike 2-space at each point. By the well-known Frobenius theorem (see e.g. Westenholz [11], Proposition 3.13), these 2-spaces admit local integral manifolds if (and only if) the Lie bracket of $L^{+}$and $L^{-}$is a linear combination of $L^{+}$and $L^{-}$. The condition of $L^{+}$and $L^{-}$being geodesic makes sure that these integral manifolds are timelike photon 2 -surfaces. This proves the 'if'-part. To prove the 'only if'-part one just has to verify that the lightlike and geodesic vector fields $l^{+}$and $l^{-}$, given on each leave of the foliation up to transformations (15), can be chosen such that they make up two smooth vector fields $L^{+}$and $L^{-}$on some neighborhood of $p$.

In Subsections 5.2 and 5.3 below we use Proposition 2 for constructing families of photon 2-surfaces in Schwarzschild, Reissner-Nordström and Gödel spacetime. In more complicated spacetimes, however, it is rather difficult to find out whether or not two vector fields $L^{+}$and $L^{-}$with the desired properties do exist. In some cases of interest it could be of help to use the Newman-Penrose formalism, in particular the modified version of this calculus, due to Geroch, Held and Penrose [12], where a pair of real lightlike vectors is picked out at each point. 
As an alternative to Proposition 2 we will now give a construction method for timelike photon 2-surfaces in Lorentzian manifolds which admit a conformal Killing vector field. To work this out, we first recall that, for every vector field $K$ on an arbitrary Lorentzian manifold, the 3 -form

$$
\omega=\beta \wedge d \beta
$$

measures the twist (or vorticity) of $K^{\prime} s$ integral curves, where the one-form $\beta$ is defined as

$$
\beta=g(K, \cdot)
$$

and $\wedge$ denotes the antisymmetrized tensor product. This observation is based on the fact (see e.g. Westenholz [11], pp. 212) that, on every simply connected region of $M, \beta$ admits an integrating factor,

$$
\beta=f d h
$$

with some scalar functions $f$ and $h$, if and only if $\omega$ vanishes. If $K$ (and thus $\beta$ ) has no zeros, (25) expresses the fact that the integral curves of $K$ are orthogonal to hypersurfaces $h=$ const. After these preparations we are now ready to prove the following proposition.

Proposition 3. Let $(M, g)$ be a Lorentzian manifold and $K$ be a conformal Killing vector field on $M$,

$$
L_{K} g=\phi g
$$

where $L_{K}$ denotes the Lie derivative in the direction of $K$ and $\phi$ is some scalar function on $M$. Define $\beta$ and $\omega$ according to (24) and (23). Let I be an open interval and $\mu: I \longrightarrow M$ a lightlike geodesic such that

$$
\omega\left(K_{\mu(s)}, \dot{\mu}(s), \cdot\right)=0
$$

and

$$
g\left(K_{\mu(s)}, \dot{\mu}(s)\right) \neq 0
$$

for all $s \in I$. Define $\Sigma$ as the set of all points in $M$ that can be connected to $\mu$ by an integral curve of $K$. Then $\Sigma$ is a timelike photon 2-surface.

Proof. Condition (28) makes sure that $K_{\mu(s)}$ and $\dot{\mu}(s)$ are linearly independent, so $\Sigma$ is a 2-dimensional submanifold of $M$. ( $\Sigma$ might fail to be an imbedded submanifold because $\mu$ or an integral curve of $K$ might be closed or almost closed.) Let $k$ denote the restriction of $K$ to $\Sigma$ and define a vector field $l^{+}$on $\Sigma$ by

$$
\begin{gathered}
{\left[l^{+}, k\right]=0,} \\
l_{\mu(s)}^{+}=\dot{\mu}(s) \quad \text { for all } \quad s \in I .
\end{gathered}
$$

As the flow of a conformal Killing vector field maps lightlike geodesics onto lightlike geodesics, $l^{+}$is lightlike and geodesic on all of $\Sigma$,

$$
g\left(l^{+}, l^{+}\right)=0 \quad \text { and } \quad \nabla_{l^{+}} l^{+}=q l^{+}
$$


with some scalar function $q$ on $\Sigma$. Moreover, (26) and (29) imply that

$$
k g\left(k, l^{+}\right)=\phi g\left(k, l^{+}\right),
$$

which, together with (28), makes sure that

$$
g\left(k, l^{+}\right) \neq 0
$$

on all of $\Sigma$. With (33) being established, the vector field

$$
l^{-}=\frac{g(k, k)}{g\left(k, l^{+}\right)^{2}} l^{+}-\frac{2}{g\left(k, l^{+}\right)} k
$$

is well-defined on $\Sigma$. Clearly, $l^{-}$is lightlike and linearly independent of $l^{+}$. This implies that $\Sigma$ is timelike. What remains to be shown is that $l^{-}$is geodesic. To prove this, we first evaluate our assumption (27). Together with (26) and (29), this assumption guarantees that

$$
\omega\left(k, l^{+}, \cdot\right)=0
$$

on all of $\Sigma$. Hence, we have for all vector fields $Y$ along $\Sigma$ (which need not be tangent to $\Sigma$ )

$$
\begin{aligned}
0= & g(k, k) d \beta\left(l^{+}, Y\right)+g\left(k, l^{+}\right) d \beta(Y, k)+g(k, Y) d \beta\left(k, l^{+}\right) \\
= & g(k, k)\left(g\left(\nabla_{l^{+}} k, Y\right)-g\left(\nabla_{Y} k, l^{+}\right)\right)+g\left(k, l^{+}\right)\left(g\left(\nabla_{Y} k, k\right)-g\left(\nabla_{k} k, Y\right)\right) \\
& +g(k, Y)\left(g\left(\nabla_{k} k, l^{+}\right)-g\left(\nabla_{l^{+}} k, k\right)\right) \\
= & g(k, k)\left(2 g\left(\nabla_{l^{+}} k, Y\right)-\phi g\left(Y, l^{+}\right)\right)-2 g\left(k, l^{+}\right) g\left(\nabla_{k} k, Y\right) \\
& +2 g(k, Y) g\left(\nabla_{k} k, l^{+}\right),
\end{aligned}
$$

where, in the last step, (26) was used. As (36) holds for all $Y$ along $\Sigma$, we have found that

$$
\begin{aligned}
0 & =2 g(k, k) \nabla_{l^{+}} k-\phi g(k, k) l^{+}-2 g\left(k, l^{+}\right) \nabla_{k} k \\
& +2 \phi g\left(k, l^{+}\right) k-2 g\left(k, \nabla_{k} l^{+}\right) k
\end{aligned}
$$

along $\Sigma$; this equation will be used later . - We now calculate $\nabla_{l^{-}} l^{-}$from (34). Using repeatedly (26), (29) and (31) we find

$$
\begin{aligned}
\nabla_{l^{-}} l^{-}= & \frac{g(k, k)}{g\left(k, l^{+}\right)^{2}}\left(\left(l^{+} \frac{g(k, k)}{g\left(k, l^{+}\right)}\right) \frac{l^{+}}{g\left(k, l^{+}\right)}-2 \nabla_{l^{+}} \frac{k}{g\left(k, l^{+}\right)}\right) \\
& -\frac{2}{g\left(k, l^{+}\right)}\left(\frac{g(k, k)}{g\left(k, l^{+}\right)} \nabla_{k} \frac{l^{+}}{g\left(k, l^{+}\right)}-2 \nabla_{k} \frac{k}{g\left(k, l^{+}\right)}\right) \\
= & \left(l^{+} \frac{g(k, k)}{g\left(k, l^{+}\right)}+\frac{2 k g\left(k, l^{+}\right)}{g\left(k, l^{+}\right)}\right) \frac{l^{-}}{g\left(k, l^{+}\right)} \\
& +\frac{4}{g\left(k, l^{+}\right)^{3}}\left(g\left(k, \nabla_{l^{+}} k\right) k-g(k, k) \nabla_{l^{+}} k+g\left(k, l^{+}\right) \nabla_{k} k\right) .
\end{aligned}
$$

By (34) and (37), this demonstrates that $\nabla_{l^{-}} l^{-}$is a multiple of $l^{-}$. 
This proposition applies, in particular, to conformally static spacetimes, i.e., to spacetimes admitting a timelike conformal Killing vector field that is hypersurface-orthogonal. If $K$ is hypersurface-orthogonal, $\omega$ vanishes, so (27) is automatically satisfied. If $K$ is timelike, $(28)$ is automatically satisfied. Hence, in a conformally static spacetime Proposition 3 associates with every lightlike geodesic a timelike photon 2-surface. In combination with Theorem 1, this gives the following observation which is exemplified by the work of Abramowicz mentioned in the introduction. In a static or conformally static spacetime, one does not feel an inertial force directed sidewards if one moves along the spatial path of a light ray.

We emphasize that (27) is automatically satisfied whenever $K$ is hypersurfaceorthogonal, independent of the causal character of $K$. Note, however, that (28) gives a (mild) restriction on the allowed lightlike geodesics $\mu$ if $K$ is not timelike. Taking this restriction into account, we can apply Proposition 3, e.g., to the spacelike and hypersurface-orthogonal Killing vector field $\partial_{\varphi}$ in a static and axisymmetric spacetime.

If $K$ is not hypersurface-orthogonal, (27) is a severe restriction on the allowed lightlike geodesics $\mu$. In a stationary but non-static spacetime only those lightlike geodesics $\mu$ give rise to a timelike photon 2 -surface for which $\dot{\mu}(s)$ points in the direction of the local rotation axis of the timelike Killing vector field for all parameter values $s$.

One might be tempted to conjecture that all timelike photon 2-surfaces can be constructed with the help of conformal Killing vector fields via Proposition 3 , i.e., that timelike photon 2 -surfaces do not exist in spacetimes without conformal Killing vector fields. We will now prove some results which demonstrate that this conjecture is false. To that end we have to characterize timelike photon surfaces in terms of their second fundamental form. Recall that the second fundamental form (or shape tensor field) $\Pi$ is well-defined for any nowhere lightlike submanifold $\Sigma$ of a semi-Riemannian manifold, in particular for a timelike submanifold of a Lorentzian manifold, by the equation

$$
\Pi(u, w)=P^{\perp}\left(\nabla_{u} w\right),
$$

where $P^{\perp}$ denotes the tensor field along $\Sigma$ which is defined by the orthogonal projections (10) and $u$ and $w$ are vector fields on $\Sigma$. As $[u, w]$ must be tangent to $\Sigma$, it is easy to verify the well-known fact that $\Pi$ is a symmetric tensor field along $\Sigma$. For our purpose, the following notion is of particular interest, cf., e.g., O'Neill [13], p. 106.

Definition 2. A nowhere lightlike submanifold $\Sigma$ of a semi-Riemannian manifold $(M, g)$ is called totally umbilic if there is a normal vector field $Z$ along $\Sigma$ such that

$$
\Pi(u, w)=g(u, w) Z
$$

for all vector fields $u, w$ on $\Sigma$.

A totally umbilic submanifold with $Z=0$ is called totally geodesic. It is easy to verify that the property of being totally umbilic is conformally invariant 
whereas the property of being totally geodesic is not. As a matter of fact, any totally umbilic submanifold can be locally converted into a totally geodesic one by a suitable conformal transformation. Totally geodesic timelike 2-surfaces have found a lot of interest since Vickers [14] and others demonstrated that the worldsheet of a self-gravitating cosmic string must be totally geodesic. Apparently there is not much interest among relativists in totally umbilic timelike 2-surfaces up to now.

We will now prove that a 2-dimensional timelike submanifold of a Lorentzian manifold is a photon 2-surface if and only if it is totally umbilic. For timelike hypersurfaces, i.e., for the case $\operatorname{dim}(M)=3$, the same result can be deduced from Claudel, Virbhadra and Ellis [10], Theorem 2.2.

Proposition 4. Let $\Sigma$ be a 2-dimensional timelike submanifold of a Lorentzian manifold $(M, g)$. Then $\Sigma$ is a photon 2-surface if and only if $\Sigma$ is totally umbilic.

Proof. Let $l^{+}$and $l^{-}$denote the two lightlike vector fields on $\Sigma$, unique up to transformations of the form (15), which are normalized according to (14). If $\Sigma$ is totally umbilic, (40) requires that $\Pi\left(l^{ \pm}, l^{ \pm}\right)=0$. By (39) and (17), this implies that $l^{+}$and $l^{-}$are geodesic, so $\Sigma$ is a photon 2-surface. - Conversely, assume that $\Sigma$ is a photon 2-surface. Consider any two vector fields $u=\alpha l^{+}+\beta l^{-}$ and $w=\gamma l^{+}+\delta l^{-}$on $\Sigma$, where $\alpha, \beta, \gamma, \delta$ are scalar functions on $\Sigma$. As $\nabla_{l^{+}} l^{+}$ and $\nabla_{l^{-}} l^{-}$are tangent to $\Sigma$, the second fundamental form (39) reduces to

$$
\Pi(u, w)=\alpha \delta P^{\perp}\left(\nabla_{l^{+}} l^{-}\right)+\beta \gamma P^{\perp}\left(\nabla_{l^{-}} l^{+}\right) .
$$

The fact that with $l^{+}$and $l^{-}$also the Lie bracket $\left[l^{+}, l^{-}\right]$must be tangent to $\Sigma$ implies

$$
P^{\perp}\left(\nabla_{l^{+}} l^{-}\right)=P^{\perp}\left(\nabla_{l^{-}} l^{+}\right)=:-2 Z .
$$

As $g(u, w)=-2 \alpha \delta-2 \beta \gamma$, (41) now demonstrates that $\Pi$ has, indeed, the form (40).

Based on this proposition we can now address the question of whether a spacetime can be foliated into photon 2-surfaces. For Riemannian manifolds (i.e., for positive definite metrics) it is known that even locally the existence of a foliation into totally umbilic submanifolds is not guaranteed; criteria in terms of curvature conditions have been given by Walschap [16]. Although, as far as we know, analogous results have not been worked out for Lorentzian or other indefinite signatures, the Riemannian results clearly indicate that only special classes of spacetimes admit a foliation into photon 2-surfaces. An interesting example is the class of twisted products. For local considerations, this class can be defined in the following way. An $n$-dimensional pseudo-Riemannian manifold is locally a twisted product if it admits local coordinates $(u, v)=\left(u^{1}, \ldots, u^{m}, v^{1}, \ldots, v^{n-m}\right)$ such that the metric takes the form

$$
g=h_{i j}(u) d u^{i} d u^{j}+\psi(u, v) k_{\mu \nu}(v) d v^{\mu} d v^{\nu}
$$

with summation over $i, j$ from 1 to $m$ and over $\mu, \nu$ from 1 to $n-m$. In the more special case that the "twisting function" $\psi$ is independent of $v$ one speaks 
of a warped product, cf. O'Neill [13]. It is an elementary exercise to verify that for any pseudo-Riemannian metric of the form (43) the submanifolds $u=$ const. are totally umbilic and the submanifolds $v=$ const. are totally geodesic. More generally, the following result is true. A pseudo-Riemannian manifold is locally a twisted product if and only if it locally admits two foliations $\mathcal{F}$ and $\mathcal{G}$ which are transverse and orthogonal to each other with all leaves of $\mathcal{F}$ totally geodesic and all leaves of $\mathcal{G}$ totally umbilic. For a proof we refer to Ponge and Reckziegel [15]; our statement is the local version of their Theorem 1. As a conformal factor has no influence upon the property of being totally umbilic, this observation together with Proposition 4 gives us the following result. Every spacetime that is conformally related to a twisted product of a Riemannian factor and a 2dimensional Lorentzian factor is foliated into timelike photon 2-surfaces. As a twisted product need not admit any non-zero conformal Killing vector field, this demonstrates the existence of timelike photon 2-surfaces that cannot be constructed by the method of Proposition 3.

\section{$5 \quad$ Examples}

\subsection{Conformally flat spacetimes}

As a photon 2-surface remains a photon 2-surface if the spacetime metric is conformally transformed, the photon 2-surfaces in a conformally flat spacetime are locally the same as the photon 2-surfaces in Minkowski spacetime.

To determine all timelike photon 2-surfaces in $n$-dimensional Minkowski spacetime we begin with the case $n=3$. O'Neill [13], p. 117, proves that a connected, complete and nowhere lightlike hypersurface in a vector space with non-degenerate scalar product of arbitrary signature is totally umbilic if and only if it is either a hyperplane or a hyperquadric. By Proposition 4, this result implies that the connected and complete timelike photon 2 -surfaces in 3-dimensional Minkowski spacetime are the timelike planes and the timelike quadrics. The latter are all timelike rotational hyperboloids, given in arbitrary Minkowski coordinates $(x, y, t)$ by an equation of the form

$$
\left(x-x_{0}\right)^{2}+\left(y-y_{0}\right)^{2}-\left(t-t_{0}\right)^{2}=r^{2},
$$

where $x_{0}, y_{0}, t_{0}$ and $r>0$ are real numbers, see Figure 1, left. A timelike plane can, of course, be interpreted as the worldsheet of a straight track in inertial motion. A timelike rotational hyperboloid can be interpreted as the worldsheet of a circular track whose radius contracts and then re-expands in the course of time. Note that, owing to the invariance of (44) with respect to Lorentz boosts, this interpretation is independent of the choice of a particular inertial system. It is obvious, intuitively, that someone moving along a track whose worldsheet is a plane does not feel centrifugal or Coriolis forces, and that the track appears straight to the eye and when probed with a gyroscope. Our results show the less obvious fact that the same is true for a track whose worldsheet is a rotational hyperboloid, and for no other tracks in 3-dimensional Minkowski spacetime. 

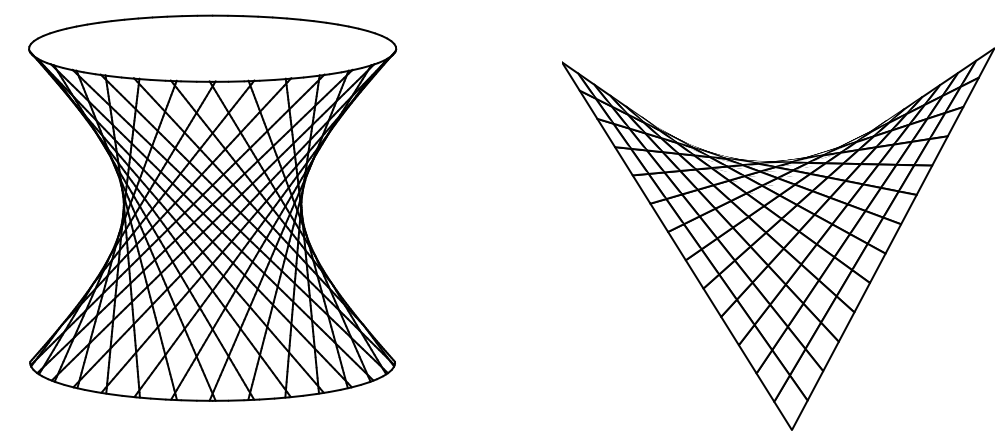

Figure 1: In affine 3-space, the only surfaces other than planes that admit two different rulings by straight lines are rotational hyperboloids (left) and hyperbolic paraboloids (right). In 3-dimensional Minkowski spacetime, the hyperbolic paraboloid cannot be realized with lightlike rulings.

By adding more spatial dimensions, a photon 2-surface in 3-dimensional Minkowski spacetime can, of course, also be interpreted as a photon 2-surface in $n$-dimensional Minkowski spacetime for $n>3$. This construction gives, indeed, all photon 2-surfaces in $n$-dimensional Minkowski spacetime for arbitrarily large $n$. In order to prove this one has to demonstrate that a totally umbilic timelike 2 -surface in $n$-dimensional Minkowski spacetime must be completely contained in a 3-dimensional affine subspace. This demonstration can be found in a paper by Hong [17]. Related results for pseudo-Euclidean spaces of arbitrary signature are given by Ahn, Kim and Kim [18].

Note that a timelike plane in Minkowski spacetime can be constructed by applying a timelike translation to a lightlike straight line. Similarly, a timelike rotational hyperboloid can be constructed by applying a spatial rotation to a lightlike straight line. Hence, all photon 2-surfaces in conformally flat spacetimes can be constructed by the method of Proposition 3.

In addition, it is interesting to mention that a photon 2 -surface in $n$-dimensional Minkowski spacetime is, in particular, a surface in $n$-dimensional affine space that admits two different rulings by straight lines. It is well known that in 3-dimensional affine space the only surfaces with this property are planes, rotational hyperboloids and hyperbolic paraboloids, see Figure 1; for a discussion of this classical result the reader may consult Hilbert and Cohn-Vossen [20]. This observation gives an alternative way of determining all timelike photon 2-surfaces in 3-dimensional Minkowski spacetime (and thereupon, by the above argument, in $n$-dimensional Minkowski spacetime): One just has to single out those doubly ruled surfaces for which both rulings are realized by lightlike lines. It is an elementary exercise to verify that the only surviving surfaces are timelike 
planes and timelike rotational hyperboloids.

\subsection{Schwarzschild and Reissner-Nordström spacetimes}

In this subsection we consider $(2+1)$-dimensional spacetimes with metrics of the form

$$
g=-e^{2 \Phi(r)} d t^{2}+e^{-2 \Phi(r)} d r^{2}+r^{2} d \varphi^{2} .
$$

This includes, as particular examples, the restriction to the equatorial plane of the Schwarzschild spacetime,

$$
e^{2 \Phi(r)}=1-\frac{2 M}{r}
$$

and of the Reissner-Nordström spacetime,

$$
e^{2 \Phi(r)}=1-\frac{2 M}{r}+\frac{Q^{2}}{r^{2}} .
$$

As both $\partial_{t}$ and $\partial_{\varphi}$ are hypersurface-orthogonal Killing vector fields, Proposition 3 allows to construct $\partial_{t}$-invariant photon 2 -surfaces and $\partial_{\varphi}$-invariant photon 2surfaces. The former are associated with static tracks, the latter with circular tracks that contract or expand in the course of time. Here it is our goal to determine the $\partial_{\varphi}$-invariant photon 2-surfaces. Instead of using Proposition 3, we find it more convenient to construct these photon 2-surfaces with the help of Proposition 2.

As an ansatz, we consider two vector fields

$$
L^{ \pm}=A(r)\left(\partial_{t}+X(r) \partial_{r}\right) \pm \frac{1}{r} \partial_{\varphi},
$$

where $A$ and $X$ are functions of $r$ to be specified later. The Lie bracket of these vector fields satisfies

$$
\left[L^{+}, L^{-}\right]=\frac{A(r) X(r)}{r}\left(L^{+}-L^{-}\right),
$$

which demonstrates that $L^{+}$and $L^{-}$are surface-forming for any choice of $A$ and $X$. The additional condition

$$
X(r)^{2}=e^{2 \Phi(r)}\left(e^{2 \Phi(r)}-A(r)^{-2}\right)
$$

makes sure that $L^{+}$and $L^{-}$are lightlike. This implies that the 2-dimensional integral manifolds generated by $L^{+}$and $L^{-}$are timelike. As each of these integral manifolds is obviously invariant under the flow of $\partial_{\varphi}$, it can be interpreted as the worldsheet of a circular track whose radius changes with time. We now add the condition that $L^{+}$and $L^{-}$be geodesic,

$$
\nabla_{L^{ \pm}} L^{ \pm}=f_{ \pm} L^{ \pm}
$$


with some functions $f_{+}$and $f_{-}$. This is true if and only if

$$
g\left(\nabla_{L^{ \pm}} L^{ \pm}, \partial_{i}\right)=f_{ \pm} g\left(L^{ \pm}, \partial_{i}\right)
$$

for $i=\varphi, t, r$. As $L^{ \pm}$is lightlike, we have

$$
g\left(\nabla_{L^{ \pm}} L^{ \pm}, \partial_{i}\right)=L^{ \pm} g\left(L^{ \pm}, \partial_{i}\right)-g\left(L^{ \pm},\left[L^{ \pm}, \partial_{i}\right]\right) .
$$

With (53) it is easy to evaluate (52). For $i=\varphi$ and $i=t$ we find respectively

$$
\begin{gathered}
A(r) X(r)=f_{ \pm} r \\
X(r) A(r) A^{\prime}(r)-2 X(r) A(r)^{2} \Phi^{\prime}(r)=f_{ \pm} A(r) .
\end{gathered}
$$

The equation for $i=r$ turns out to be a consequence of (50), (54) and (55), so we do not have to consider it. Upon eliminating $f_{ \pm}$from (54) and (55) we get an ordinary first-order differential equation for $A$ which can be solved easily, yielding

$$
A(r)=\frac{r}{R} e^{-2 \Phi(r)},
$$

where, without loss of generality, the constant of integration $R$ can be assumed positive. For any choice of $R(>0)$, inserting (56) into (50) gives us a positive and a negative solution $X$ on that part of the spacetime where

$$
r^{2} e^{-2 \Phi(r)}>R^{2} .
$$

If (57) holds on the whole spacetime under consideration, this construction gives us two families of photon 2-surfaces, one corresponding to the positive solution for $X$ and one corresponding to the negative solution for $X$, such that the members of either family foliate the spacetime. If (57) is true only on a proper subset $U$ of the spacetime, then it is not difficult to verify that all members of both families either meet the boundary $\partial U$ tangentially or asymptotically approach the boundary $\partial U$. In the first case each member of the first family is to be glued together with a member of the second family at the boundary. So there is actually only one family of photon 2-surfaces which covers $U$ twice.

These results are best illustrated with the exterior Schwarzschild spacetime. To that end we specify $\Phi$ according to (46) and we restrict to the region $r>2 M$. For $R<\sqrt{27} M,(57)$ is satisfied for all $r>2 M$. Hence, $R$ is associated with two different families of photon 2-surfaces. Each of these photon 2-surfaces is generated by lightlike geodesics that extend from the horizon at $r=2 \mathrm{M}$ to infinity, see Figure 2, top. For $R=\sqrt{27} M$, (57) is true everywhere on $r>2 M$ with the exception of the value $r=3 M$ where the left-hand side of (57) is equal to the right-hand side. It is still true that $R$ is associated with two different families of photon 2-surfaces; however, the surface $r=3 \mathrm{M}$ belongs to both families. All other members of both families asymptotically approach this particular photon 2-surface at $r=3 M$ either for $t \rightarrow \infty$ or for $t \rightarrow-\infty$, see Figure 2, middle. For $R>\sqrt{27} M,(57)$ is true for all $r>2 M$ with the exception of some interval around $r=3 \mathrm{M}$. There is only one family of photon 2 -surfaces 

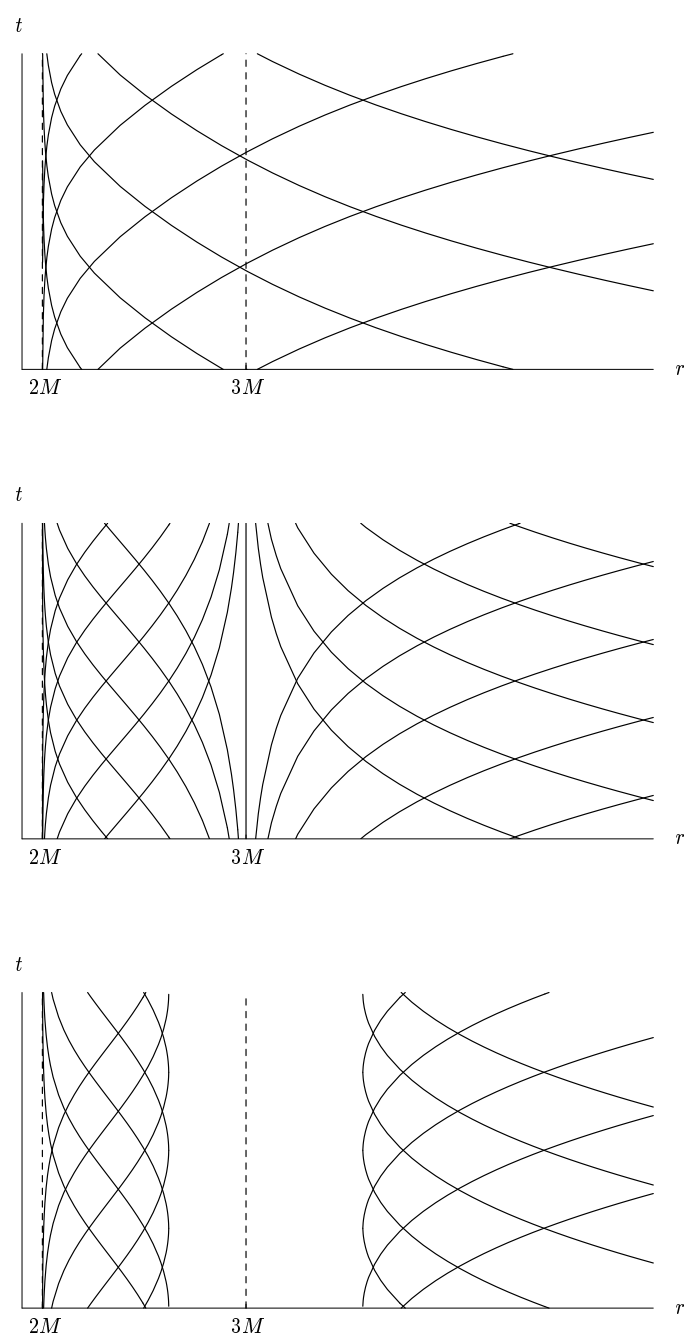

Figure 2: $\partial_{\varphi}$-invariant timelike photon 2-surfaces in Schwarzschild spacetime 
associated with $R$ which covers the allowed region twice. Each member of this family is ruled by lightlike geodesics which either come from infinity, reach a minimal radius and go out to infinity, or come from the horizon, reach a maximal radius and go down to the horizon, see Figure 2, bottom.

\subsection{Gödel spacetime}

The Gödel metric

$$
g=-d t^{2}+d x^{2}-\frac{1}{2} e^{2 \sqrt{2} \omega_{o} x} d y^{2}+d z^{2}-2 e^{\sqrt{2} \omega_{o} x} d t d y
$$

is a rotating dust solution of Einstein's field equation with cosmological constant, cf., e.g., Hawking and Ellis [19], p. 168. A foliation of the Gödel spacetime into timelike photon 2-surfaces can be easily found with the help of Proposition 2. To that end, we observe the obvious fact that the vector fields

$$
L^{ \pm}=\partial_{t} \pm \partial_{z}
$$

are lightlike and have vanishing Lie bracket, $\left[L^{+}, L^{-}\right]=0$. Moreover, for all basis vector fields $\partial_{i}$ with $i=t, z, x, y$ we read from (58)

$$
g\left(\nabla_{L^{ \pm}} L^{ \pm}, \partial_{i}\right)=L^{ \pm} g\left(L^{ \pm}, \partial_{i}\right)-\frac{1}{2} \partial_{i}\left(g\left(L^{ \pm}, L^{ \pm}\right)\right)=0
$$

so $\nabla_{L^{ \pm}} L^{ \pm}=0$. By Proposition 2 , the surfaces $x=$ const., $y=$ const. are timelike photon 2-surfaces. At the same time, these timelike photon 2 -surfaces exemplify Proposition 3 , where $K$ is to be identified with the Killing vector field $\partial_{t}$. To apply Proposition 3 one just has to verify from (58) that for $\beta=g\left(\partial_{t}, \cdot\right)$ the twist 3-form $\omega=\beta \wedge d \beta$ satisfies $\omega\left(\partial_{t}, \partial_{z}, \cdot\right)=0$; thus, (27) is indeed true for $\dot{\mu}$ parallel to $L^{+}$or $L^{-}$.

It is interesting to note that this foliation into timelike photon 2-surfaces of the Gödel spacetime is not associated with a twisted-product structure. This is obvious from the fact that, because of the $d y d t$-term in the metric (58), the 2 -spaces orthogonal to the surfaces $x=$ const., $y=$ const. are not integrable.

The surfaces $x=$ const., $y=$ const. are not the only timelike photon 2 surfaces in the Gödel cosmos. Other examples can be found, e.g., by applying Proposition 3 to the hypersurface-orthogonal spacelike Killing vector field $K=$ $\partial_{z}$ and to any lightlike geodesic $\mu$ that is not perpendicular to $\partial_{z}$.

\section{References}

[1] Abramowicz M A, Carter B, and Lasota J P 1988 Gen. Rel. Grav. 201173

[2] Abramowicz M A and Prasanna A R 1990 Mon. Not. R. Astr. Soc. 245 720

[3] Abramowicz M A 1990 Mon. Not. R. Astr. Soc. 245733 
[4] Abramowicz M A and Sonego S The optical theory of black holes (Singapore: World Scientific) to appear

[5] Abramowicz M A, Nurowski P, and Wex N 1993 Class. Quantum Grav. 10 L183

[6] de Felice F 1991 Mon. Not. R. Astr. Soc. 252197

[7] Sonego S and Massar M 1996 Class. Quantum Grav. 13139

[8] Bini D, Carini P and Jantzen R T 1997 Int. Journ. Mod. Physics D 61

[9] Misner C, Thorne K and Wheeler J 1973 Gravitation (San Francisco: Freeman)

[10] Claudel C M, Virbhadra K S, and Ellis G 2001 J. Math. Phys. 42818

[11] Westenholz C v 1978 Differential forms in mathematical physics (Amsterdam: North-Holland)

[12] Geroch R, Held A and Penrose R 1973 J. Math. Phys. 14874

[13] O'Neill B 1983 Semi-Riemannian Geometry (New York: Academic Press)

[14] Vickers J A G 1987 Class. Quantum Grav. 41

[15] Ponge R and Reckziegel H 1993 Geometriae Dedicata 4815

[16] Walschap G 1997 Ill. J. Math. 41122

[17] Hong S-K 1997 Bull. Korean Math. Soc. 349

[18] Ahn S-S, Kim D-S, and Kim Y H 1996 J. Korean Math. Soc. 33507

[19] Hawking S W and Ellis G F R 1973 The large scale structure of space-time (Cambridge: Cambridge Univ. Press)

[20] Hilbert D and Cohn-Vossen S 1991 Geometry and the imagination (Providence: AMS Chelsea) 